\title{
Losartan inhibits conventional dendritic cell maturation and Th1 and Th17 polarization responses: Novel mechanisms of preventive effects on lipopolysaccharide-induced acute lung injury
}

\author{
JUN LIU, PENG-SHU ZHANG, QING YU, LING LIU, YI YANG, FENG-MEI GUO and HAI-BO QIU \\ Department of Critical Care Medicine, Nanjing Zhong-Da Hospital, Southeast University \\ School of Medicine, Nanjing 210009, P.R. China
}

Received August 18, 2011; Accepted September 28, 2011

DOI: $10.3892 / \mathrm{ijmm} .2011 .818$

\begin{abstract}
Acute lung injury (ALI) remains one of major causes of morbidity and mortality in intensive care medicine. The lack of efficient pharmacological interventions contributes to the high mortality rate of ALI. Losartan, an antagonist of angiotensin II (Ang II) type 1 receptor, is a potent therapeutic drug for ALI. Recent reports suggest that losartan inhibits the maturation of dendritic cells (DCs), impairs T-helper (Th) 1 immune response and ultimately attenuates inflammation in several Ang II-mediated inflammatory diseases. However, the possible protective mechanisms of losartan in ALI remain poorly understood. This study was aimed to define the effect of losartan on the frequency and phenotype of respiratory conventional DCs (cDCs) and Th cells polarization in lipopolysaccharide (LPS)-induced ALI mice. Results demonstrate that early after induction of LPS-induced ALI, cDCs expressing modest amounts of CD80 rapidly accumulated in the lungs. In addition, polarized Th1 and Th17 responses were markedly increased in LPS-induced ALI mice at 24 and $48 \mathrm{~h}$. Of note, losartan led to inhibition of respiratory cDCs maturation at $6 \mathrm{~h}$ and suppressed Th1 and Th17 polarization responses compared with ALI mice at 24 and $48 \mathrm{~h}$. Collectively, our findings may provide a novel and, at least, partial explanation for the protective effects by which losartan inhibits lung cDCs maturation and suppresses Th1 and Th17 immune responses.
\end{abstract}

Correspondence to: Professor Hai-Bo Qiu, Department of Critical Care Medicine, Nanjing Zhong-Da Hospital, Southeast University School of Medicine, 87 Dingjiaqiao Road, Nanjing 210009, P.R. China E-mail: haiboq2000@yahoo.com.cn

Abbreviations: ALI, acute lung injury; Ang II, angiotensin II; ARDS, acute respiratory distress syndrome; cDCs, conventional dendritic cells; DCs, dendritic cells; FCM, flow cytometry; i.t., intratracheal instillation; LPS, lipopolysaccharide; mDC, myeloid dendritic cell; pDC, plasmacytoid DC

Key words: acute respiratory distress syndrome, angiotensin II, dendritic cells, inflammation

\section{Introduction}

Acute lung injury (ALI) and acute respiratory distress syndrome (ARDS), characterized by an excessive inflammatory response involving a variety of inflammatory mediators, are associated with high morbidity and mortality in intensive care medicine (1). Over the past several decades, many pharmacological interventions for these entities have been evaluated, but none has clearly been shown to decrease mortality. Searching for effective therapeutic drugs is still urgently needed.

Most recently, much attention has been focused on the involvement of the renin-angiotensin system (RAS) in the pathogenesis of ALI. In particular, our laboratory and others have demonstrated that angiotensin II (Ang II), as the major effector molecule of the RAS, is significantly increased in lung tissues and plays a key pro-inflammatory role in several ALI animal models (2-5). Losartan, an antagonist of Ang II type 1 receptor, is a potent therapeutic drug for ALI $(2,5)$. However, the protective mechanisms of losartan in ALI remain poorly understood.

The pattern of immune response in ALI is incompletely characterized. Dendritic cells (DCs), including conventional DCs (cDCs) and plasmacytoid DCs (pDCs), are crucial for the priming phase of the immune response (6). DCs are widely distributed in mucosal surfaces, including the lung (7). Indeed, DCs are thought to participate in a number of inflammatory lung diseases in both humans and mice (asthma, pulmonary infections, chronic obstructive pulmonary disease) (7-9). Most recently, a study has observed that mature DCs are markedly increased in the lung and play a key pro-inflammatory role in bleomycin-induced lung injury mice (10). Therefore, control of DCs function may be critical for strategies to modulate the lung inflammatory response.

T-helper (Th) cells are known to play a significant role in the initiation of immune responses by providing help to other cells, which could at least be divided into subsets: Th1, Th2 and Th17 $(6,11)$. Two recent clinical observations have shown that severe H1N1 influenza with ARDS is characterized by early secretion of Th1 and Th17 cytokines $(12,13)$. These cytokine profiles have been previously reported to participate in both pro-inflammatory and antiviral responses. However, the exact role of Th cells in the ALI model merits further investigation. 
Recently, a number of studies have demonstrated that RAS activation could play an important role in immunologically induced inflammation (14-16). In vitro and in vivo studies have shown that Ang II could induce DCs maturation $(14,17)$. Additionally, several lines of evidence demonstrate that Ang II induces Th1 and Th17 immune responses and ultimately enhances inflammation in several Ang II-mediated inflammatory diseases (15-18). However, the direct regulatory effect of losartan on the function of lung DCs and Th cells in ALI remains to be fully elucidated. Based on these findings, therefore, we hypothesized that losartan prevented ALI in mice by inhibiting the maturation of cDCs and Th polarization response in ALI.

In the present study we used a clinically relevant model of ALI induced by intratracheal (i.t.) lipopolysaccharide (LPS) challenge in mice. One of the most interesting observations in this study is that LPS-induced ALI results in a rapid accumulation of cDCs and Th1 and Th17 polarization responses in the lungs. Of note, losartan led to inhibition of respiratory cDCs maturation and suppressed Th1 and Th17 immune responses compared with ALI mice. Cumulatively, these data show that the protective effect of losartan in ALI may be dependent, at least in part, on inhibited lung cDCs maturation and suppressed Th1 and Th17 polarization response.

\section{Materials and methods}

Animals. Male C57BL/6 mice between 6 to 10 weeks of age were obtained from the animal center of the Southeast University. The mice were housed individually in cages in a room kept at $18-24^{\circ} \mathrm{C}$ and $40-70 \%$ relative humidity, with a $12 \mathrm{~h}$ light/dark cycle. They were allowed free access to standard rodent chow and drinking water. The studies were approved by the local Institutional Animal Care and Use Committee.

Reagents. The following monoclonal antibodies (mAbs) purchased from eBioscience (San Diego, CA, USA) were used: anti-CD11c-PE (clone N418), anti-CD11b FITC (clone M1/70), anti-MHC II-APC (clone M5/114.15.2), anti-CD80-APC (clone 16-10A1), and rat anti-mouse CD16/32 (clone 93). Isotype-matched irrelevant control mAbs (all obtained from eBioscience) were tested simultaneously in all experiments. LPS from Escherichia coli strain 0111:B4 (catalog no. L2630) and collagenase type V were purchased from Sigma-Aldrich (St. Louis, MO, USA). Losartan was obtained from Cayman Chemical Company (Ann Arbor, MI, USA). RNAiso ${ }^{\mathrm{TM}}$ Plus (no. D9108A), PrimeScript ${ }^{\mathrm{TM}}$ RT reagent kit (no. DRR037A), and SYBR ${ }^{\circledR}$ Premix Ex Taq ${ }^{\mathrm{TM}}$ (no. DRR041A) were obtained from Takara Biotechnology Co., Ltd (Dalian, China). The ELISA kits for detection of mouse interleukin (IL)-6, interferon (IFN)- $\gamma$, IL-4 and IL-17 were obtained from R\&D Systems, Inc. (Minneapolis, MN, USA).

Experimental protocol. The mice were randomly assigned to one of the following three groups: i) control group (Con): mice received intraperitoneal administration of phosphate-buffered saline (PBS) $30 \mathrm{~min}$ before i.t. instillation of $30 \mu \mathrm{l}$ PBS; ii) ALI group (ALI): mice received intraperitoneal administration of PBS $30 \mathrm{~min}$ before i.t. instillation of LPS $(2 \mathrm{mg} / \mathrm{kg})$ in $30 \mu \mathrm{l}$ PBS and iii) Losartan + ALI group (Los): losartan was administered intraperitoneally $(15 \mathrm{mg} / \mathrm{kg}) 30 \mathrm{~min}$ before i.t. instillation of LPS $(2 \mathrm{mg} / \mathrm{kg})$ in $30 \mu \mathrm{l}$ PBS. The dose of losartan to carry out this experiment was chosen on the basis of prior experiments $(2,3)$. The animals were sacrificed and lungs were harvested at the following time points: 6,24 and $48 \mathrm{~h}$.

Experimental murine model of LPS-induced ALI. ALI model in mice was induced by i.t. LPS. LPS was dissolved in sterile PBS. After being anesthetized with pentobarbital sodium, each mouse was challenged i.t. in a single bolus at a dose of $2 \mathrm{mg} / \mathrm{kg}$ LPS (30 $\mu$ l PBS, room temperature). The mice were shaken vigorously to facilitate dispersion of the PBS. At varying time points after challenge, mice were sacrificed with an overdose of sodium pentobarbital.

Lung injury evaluations. ALI was evaluated by measuring the lung wet weight-to-body weight ratio (LW/BW), lung tissue histology and Smith's scores at 24 and $48 \mathrm{~h}$ after LPS or PBS challenge.

The $L W / B W$. The wet lung weight was measured as an indicator of lung inflammation (19). Before the experiment, the body weight of the animals was determined. All animals were sacrificed at indicated time points, and the lungs were immediately weighed after careful excision of extraneous tissues. The $\mathrm{LW} / \mathrm{BW}$ ratio was then calculated.

Lung histology analysis. After the animals were sacrificed, left lungs were quickly removed and processed as indicated below. The right upper lung tissues were stained with hematoxylin and eosin. A pathologist blind to the animal groups semiquantitatively evaluated the severity of lung injury according to Smith et al (20). Briefly, 10 randomly chosen regions of lung parenchyma per sample were graded on a scale of 0-4 ( 0 , absent and appears normal; 1 , light; 2 , moderate; 3 , strong; 4 , intense) for edema, inflammation, hemorrhage, necrosis and hyaline membrane formation. The lung injury was then calculated based on the mean score of the above parameters.

Lung cell isolation. To analyze lung cDCs phenotype, singlecell suspensions were isolated from the lung interstitium by enzymatic digestion. Isolation of left lung cells were performed as described in detail previously (21). Briefly, lungs were lavaged and dissected into small pieces finely using scissors, and then digested with type $\mathrm{V}$ collagenase. Then single-cell suspensions were generated by pressing digested lung through a $70-\mu \mathrm{m}$ sterile mesh, lysing in red blood cell lysis buffer, and washing in PBS.

Flow cytometry (FCM). To determine whether LPS-induced ALI altered the frequency of pulmonary cDCs, lung tissues were harvested and stained with mAb staining at $6 \mathrm{~h}$ after LPS or PBS challenge. A previously described specific staining and gating strategy by FCM has been proven to identify lung cDCs accurately in mice, and served as the basis of our approach (22).

To minimize non-specific binding, single-cell suspensions were prepared as described above, and preincubated with purified rat anti-mouse CD16/32. Then the cells were stained for $30 \mathrm{~min}$ with fluorochrome-labeled mAbs. Appropriate 
isotype-matched control mAbs were substituted for specific Abs to obtain negative controls. For each condition, at least 30,000 events were analyzed by FCM on a FACSCalibur with CellQuest software (BD Biosciences, Mountain View, CA). The frequencies of cDCs are given as a percentage of total lung cells. Thereafter, cytometer parameters and gate position were held constant during analysis of all samples.

Real-time PCR. Total-RNA extraction using RNAiso ${ }^{\mathrm{TM}}$ Plus was prepared as previously described (23). The expression levels of genes were determined by quantitative real-time reverse transcriptase-polymerase chain reaction (RT-PCR) using an ABI-Prism 7300 Sequence BioDetector (PE Biosystems, Foster City, CA). Primers for the transitional factor T-box expressed in T-cells (T-bet), GATA-binding protein-3 (GATA-3), retinoidrelated orphan receptor (ROR) $\gamma \mathrm{t}$, and the housekeeping gene $\beta$-actin were obtained from Takara Biotechnology (Dalian) Co. Ltd. Reverse transcription was performed using PrimeScript ${ }^{\mathrm{TM}}$ RT reagent kit [oligo(dt) primer and random hexamers], and cDNA amplification was performed using SYBR ${ }^{\circledR}$ Premix Ex $\mathrm{Taq}^{\mathrm{TM}}$. Primers included: T-bet, sense, 5'-ACCACCTGTTGT GGTCCAAG-3' and antisense, 5'-CACCAAGACCACATC CACAA-3'; GATA-3, sense, 5'-ACCGGGTTCGGATGTAAG TC-3' and antisense, 5'-AGGCATTGCAAAGGTAGTGC-3'; ROR $\gamma \mathrm{t}$, sense, 5'-ACGGCCCTGGTTCTCATCA-3' and antisense, 5'-CCAAATTGTATTGCAGATGTTCCAC-3'; $\beta$-actin, sense, 5'-CCTCTATGCCAACACAGTGC-3' and antisense, 5'-GTACTCCTGCTTGCTGATCC-3'.

Cycle parameters were as follows: a denaturation step at $95^{\circ} \mathrm{C}$ for $30 \mathrm{sec}$, followed by up to 40 cycles composed of $5 \mathrm{sec}$ denaturation at $95^{\circ} \mathrm{C}$ and $31 \mathrm{sec}$ annealing at $60^{\circ} \mathrm{C}$. For analysis, the fluorescence values of the threshold cycle were collected at the end of the annealing step from each reaction. The threshold cycle values obtained from $\beta$-actin amplification were used to normalize transitional factor quantification. Data are expressed as relative increase of specific mRNA as measured by fluorescence intensity of the treated samples compared with the untreated control sample, which was used as calibrator.

ELISA measurement. Lung samples were harvested at 24 and $48 \mathrm{~h}$ after PBS or LPS i.t. instillation, and lung homogenates were prepared by homogenizing previously frozen right lower lobe of lung tissue as described previously (5). Homogenates were then centrifuged at $400 \mathrm{x} \mathrm{g}$ for $10 \mathrm{~min}$ with supernatants collected and frozen at $-80^{\circ} \mathrm{C}$ until used in the assays. Levels of IL-6, IFN- $\gamma$, IL-4 and IL-17 in lung homogenates were assayed using commercially available ELISA kits, according to the manufacturer's instructions. The concentrations of these cytokines were calculated from a standard curve and expressed in picograms/milligram lung tissue.

Statistical analysis. Values for all measurements are expressed as means \pm SD. Statistical differences between experimental groups were evaluated by Student's t-test (for comparison between two samples) or by a one-way analysis of variance (for multiple comparisons). If variance was heterogeneous, group data were compared by the Mann-Whitney test for two groups' comparisons and the Tamhane's T2 test for $\mathrm{n}$ group comparison. A P-value $<0.05$ was considered to be significant.

\section{Results}

Losartan exerts a protective effect on LPS-induced ALI. The LW/BW was correlated with the severity of lung injury as previously reported (19). The LW/BW in ALI mice at 24 and $48 \mathrm{~h}$ was significantly higher than in PBS-challenged mice, indicating that LPS administration effectively induced ALI (Fig. 1A). In contrast, when ALI mice were pre-treated with losartan, the LW/BW was significantly lower than that of ALI mice at 24 and $48 \mathrm{~h}(\mathrm{P}<0.05 \mathrm{vs}$. ALI, respectively).

Then, we used histological evaluation of the lungs to confirm LPS-induced lung injury in mice. There were minimal pathological changes in the lung tissue from PBS-injected mice. On the other, the lung specimens in ALI mice at $24 \mathrm{~h}$ revealed widespread alveolar wall thicking caused by edema, striking inflammatory cell infiltration, and severe interstitial hemorrhage as previously reported (Fig. 1B) (24). In contrast, the histological changes in the losartan group were significantly improved compared with those in ALI mice at $24 \mathrm{~h}$ (Fig. 1B). As shown in Fig. 1C, quantal scoring of histological lung injury in the ALI group was markedly higher than in the normal mice at 24 and $48 \mathrm{~h}(\mathrm{P}<0.05$ vs. Con $)$. Pre-treatment of ALI mice with losartan, significantly reduced the quantal scoring of histological lung injury ( $\mathrm{P}<0.05 \mathrm{vs.} \mathrm{ALI).}$

The response to LPS was also monitored by using IL-6 as an inflammatory marker. With an i.t. LPS challenge, IL-6 levels in lung mince from ALI mice were markedly elevated compared with those from control mice at $24 \mathrm{~h}$. The concentration of IL- 6 was also modestly reduced in losartan mice compared with ALI mice at 24 and $48 \mathrm{~h}(\mathrm{P}<0.05$ vs. ALI) (Fig. 1D). Together, these data proved that early pre-treatment with losartan in ALI mice showed a protective effect on the LPS-induced ALI at both 24 and $48 \mathrm{~h}$, although lung injury was not completely prevented.

Losartan inhibits lung cDCs maturation in LPS-induced $A L I$. Among the lung DCs, two subsets have been identified, including $\mathrm{cDCs}$ and $\mathrm{pDCs}$, of which the $\mathrm{cDC}$ subset is by far the predominating DC population $(>95 \%)(25,26)$. Furthermore, respiratory cDCs are preferentially localized in the lung interstitium, and upon activation, these cells produce a range of inflammatory mediators $(26,27)$. A previous study has demonstrated that the inhalation of pathogenic material, such as bacteria or viral particles, induce a very rapid recruitment of DCs into the airways of rodents, in some cases peaking already $2 \mathrm{~h}$ after challenge (28). Therefore, to determine whether LPS-induced ALI altered the frequency of cDCs, lung tissues were harvested and stained with $\mathrm{mAb}$ staining at $6 \mathrm{~h}$ after LPS or PBS challenge.

In unchallenged mice, a distinct population of $\mathrm{CD}_{11 \mathrm{c}^{+}}$ $\mathrm{CD}_{11} \mathrm{~b}^{+} \mathrm{cDCs}$ was detectable in lung mince (Fig. 2A). The frequency of cDCs among lung tissue was $1.46 \pm 0.25 \%$ of the total cells in the lung mince at $6 \mathrm{~h}$, in agreement with basal numbers in a previous study of mice (29). In contrast, as shown in Fig. 2B, the fraction of cDCs relative to lung cells in the ALI and losartan group were significantly increased compared with those from the control mice at $6 \mathrm{~h}$, and comprised $3.39 \pm 0.74 \%$ and $2.91 \pm 0.53 \%$ of the total cells in the lung, respectively ( $\mathrm{P}<0.05$ vs. Con). This result demonstrates that total lung cDCs recruitment is rapidly increased in LPS-induced ALI mice. The 

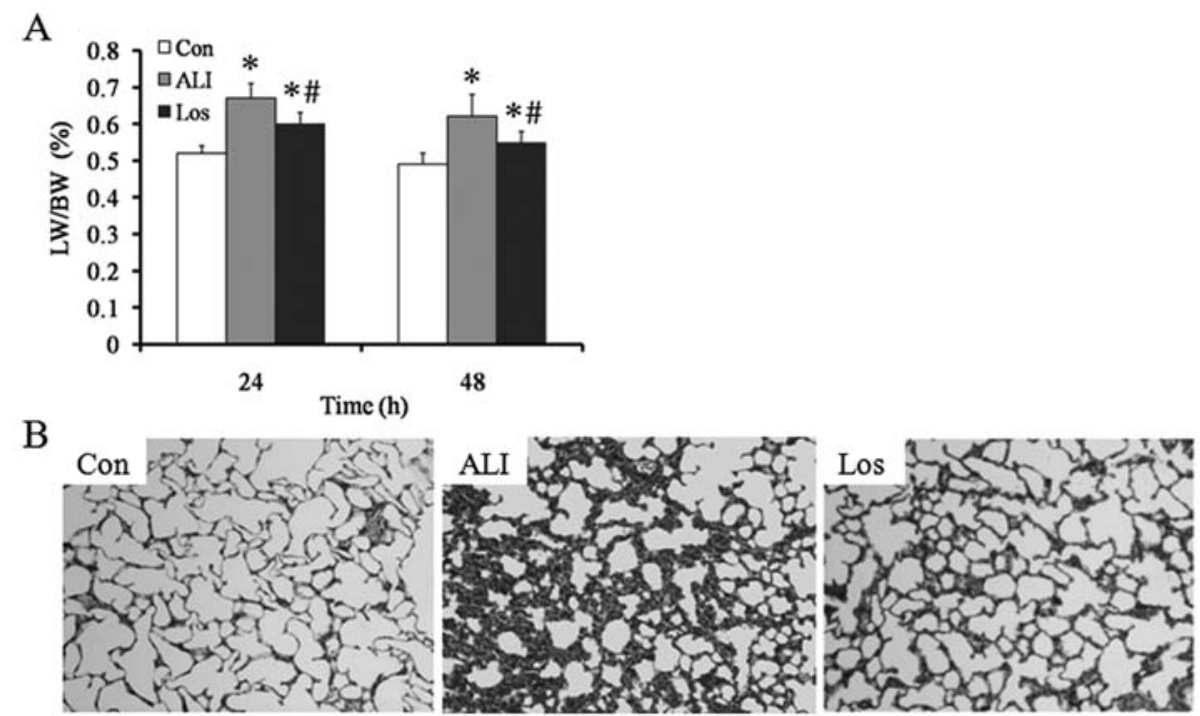

$\mathrm{C}$

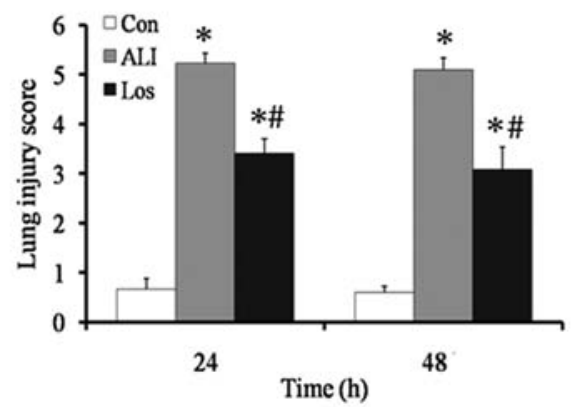

$\mathrm{D}$

Figure 1. Losartan exerts a protective effect on LPS-induced ALI. (A) Comparison of the wet lung-to-body weight ratio (LW/BW) in different groups at 24 and $48 \mathrm{~h}$. (B) Representative histology sections of lung tissue at $24 \mathrm{~h}$ (hematoxylin and eosin staining; magnification, $\mathrm{x} 100$ ). (C) The pathological lung injury score at 24 and $48 \mathrm{~h}$. (D) Comparison of IL- 6 level in the lung mince at 24 and $48 \mathrm{~h}$. These data are expressed as the mean \pm SD (n=6 for each group at the indicated time points). ${ }^{*} \mathrm{P}<0.05$ vs. the Con mice; ${ }^{~} \mathrm{P}<0.05$ vs. the ALI mice.

simultaneous accumulation of cDCs in the lungs suggests a prominent role for this DC subset in antigen presentation during LPS-induced ALI.

Mature or activated DCs are characterized by a further increase of co-stimulatory molecules (e.g., CD80, CD86, CD40) and MHC II on their surface (7). We next analyzed the maturation status of lung cDCs based on the expression of CD80 and MHC Class II. At baseline, lung cDCs in the control group at $6 \mathrm{~h}$ only expressed very low levels of the co-stimulatory molecule CD80, and comprised $3.1 \pm 1.0 \%$ of the total cells, consistent with an immature phenotype. This finding is similar to that reported by other researchers (30). In parallel to the accumulation of pulmonary cDCs in ALI mice, the expression of CD80 on the respiratory cDCs surface showed a significant increase $(10.1 \pm 2.9 \%)$. Of note, pre-treatment with losartan led to a marked reduction in CD80 expression on pulmonary cDCs $(\mathrm{P}<0.05$ vs. ALI). In contrast, the expression of MHC II was not significantly different among the groups (Fig. 2D). This result suggests that respiratory cDCs appear to have a modest maturation phenotype in ALI mice, and losartan treatment significantly inhibits lung cDCs maturation in LPS-induced ALI mice at $6 \mathrm{~h}$.

Losartan inhibits Th1 and Th17 polarization responses in $L P S$-induced ALI. As previously demonstrated, lung DCs play a key role in activating and expanding $\mathrm{CD}^{+} \mathrm{T}$ cells in the lung compartment (6). Upon receiving signals through the binding of antigen to the $\mathrm{T}$-cell receptor in the presence of polarizing cytokines, naive Th precursor cells differentiate into Th1, Th2 or Th17 effector cells $(6,11)$. However, the Th cells recruitment in LPS-induced ALI model has not been fully defined.

To assess Th polarization, real time RT-PCR was performed on lung mRNA obtained from all groups at 24 and $48 \mathrm{~h}$. The mRNA expression of the Th1-inducing transcription factor, T-bet, was up-regulated $>2$-fold in ALI mice at $24 \mathrm{~h}$ and further enhanced $>6$-fold at $48 \mathrm{~h}$ relative to normal mice $(\mathrm{P}<0.05$ vs. Con) (Fig. 3A). In contrast, there was no significant difference in the expression of GATA-3 (the specific transcription factor of Th2 cells) among groups at 24 and $48 \mathrm{~h}$ (Fig. 3C). However, the mRNA expression of ROR $\gamma \mathrm{t}$, the Th17-inducing transcription factor, was shown a slight but significant increase at 24 and $48 \mathrm{~h}$ in the lung tissues of the ALI mice ( $\mathrm{P}<0.05 \mathrm{vs}$. Con). The increase in T-bet and ROR $\gamma \mathrm{t}$ mRNA over time suggests that LPS-induced ALI led to lung Th1 and Th17 polarization responses.

Next, we evaluated the production of Th1, Th2 and Th17 cytokines in mice at 24 and $48 \mathrm{~h}$ after PBS or LPS challenge. In terms of Th1 cytokine production, the levels of IFN- $\gamma$ in lung tissue were significantly increased at 24 and $48 \mathrm{~h}$ after LPS challenge (Fig. 3B). This observation is in agreement 
A

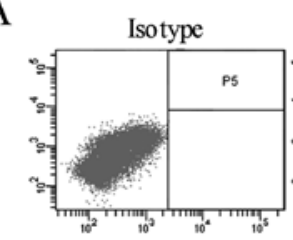

ALI

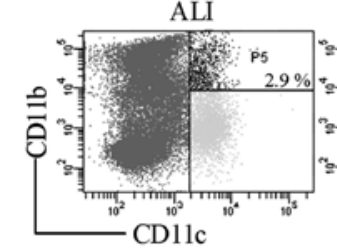

$\mathrm{C}$
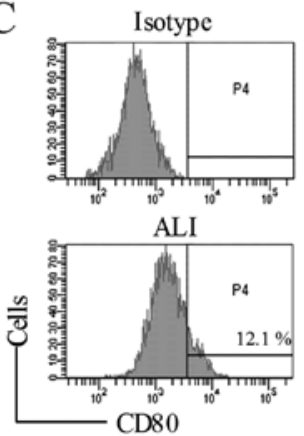

E
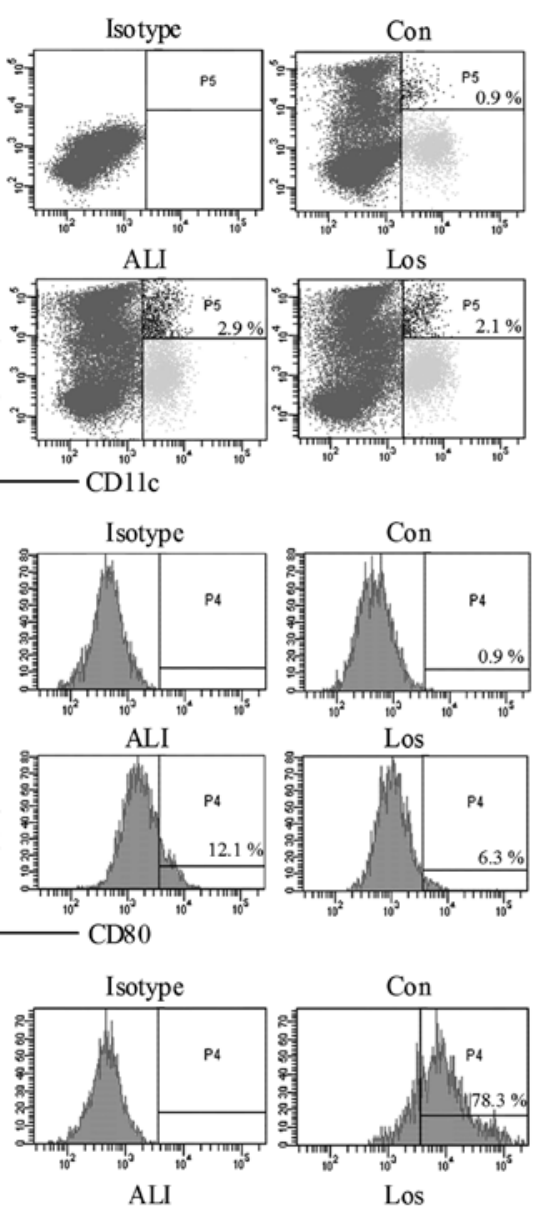

Los

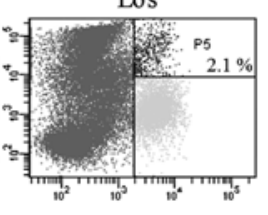

Con

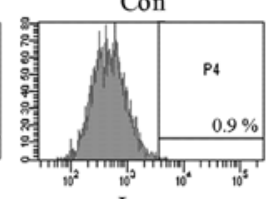

Los

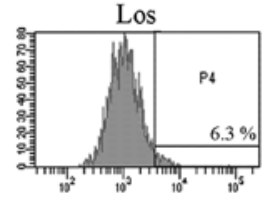

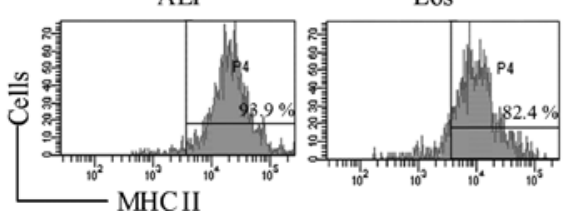

$\mathrm{F}$

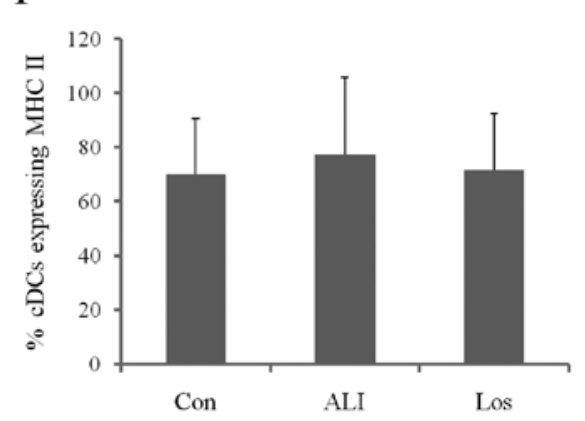

B

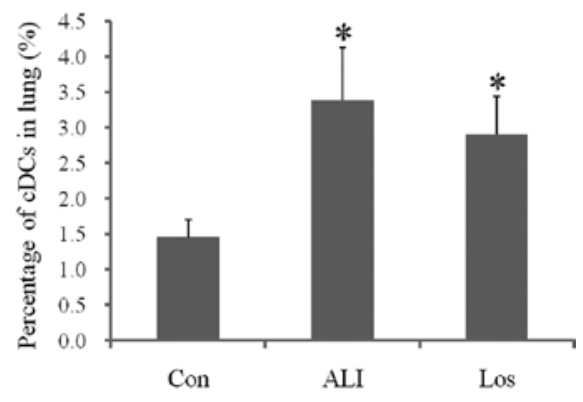

D

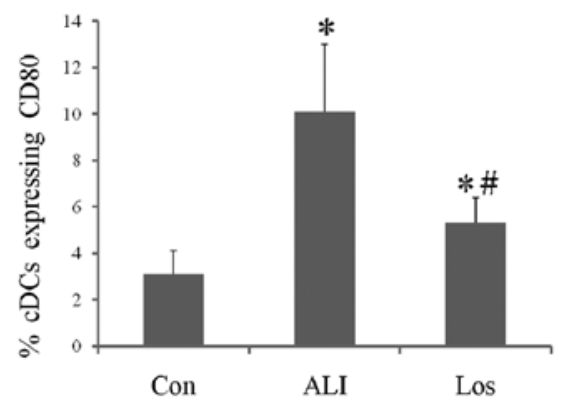

Con ALI Los

Figure 2. Losartan inhibits pulmonary cDCs maturation in LPS-induced ALI at $6 \mathrm{~h}$. (A and B) Representative dot plots and bar graph showing the percentage of the respiratory cDCs (gate on the upper right in each panel-P5) in the absence or presence of losartan. (C and D) Charts and bar graph showing the expression of CD80 on respiratory cDCs (gate on the right in each panel-P4). (E and F) Charts and bar graph showing the expression of MHC II on lung cDCs (gate on the right in each panel-P4). Data are the mean $\pm \mathrm{SD}$ ( $\mathrm{n}=11$ or 12 for each group). ${ }^{\mathrm{P}} \mathrm{P}<0.05$ vs. Con mice; ${ }^{*} \mathrm{P}<0.05$ vs. ALI mice.

with a previous report in mice given intraperitoneal endotoxin (31). In addition, lung IL-4 (a Th2 cytokine) levels were significantly enhanced at 24 and $48 \mathrm{~h}$ in the LPS-induced ALI mice (Fig. 3D). Regarding IL-17 (a signature cytokine of Th17), a significant increase was noted at 24 and $48 \mathrm{~h}$ in LPS-induced ALI mice (Fig. 3F). These findings demonstrate that both Th1 and Th17 polarization responses are important in the development of LPS-induced ALI.

We thus analyzed whether blocking AT1R suppresses the pro-inflammatory Th1 and Th17 responses during ALI. Pre-treatment of ALI mice with losartan resulted in significantly reduced mRNA expression of T-bet at 24 and $48 \mathrm{~h}$ ( $\mathrm{P}<0.05$ vs. ALI) (Fig. 3A), but did not alter the expression of GATA-3 (Fig. 3C). Finally, losartan resulted in significantly reduced ROR $\gamma \mathrm{t}$ mRNA expression at $48 \mathrm{~h}(\mathrm{P}<0.05$ vs. ALI) (Fig. 3E). In terms of the production of Th1 and Th17 cytokines, both Th1 polarizing (IFN- $\gamma$ ) and Th17 polarizing (IL-17) cytokines in lung tissue at 24 and $48 \mathrm{~h}$ decreased significantly in losartan-pre-treated mice compared with the ALI mice (Fig. 3B and F). Collectively, our results support the notion that showing the percentage of
ph showing the expression

losartan induces a regulatory phenotype capable of suppressing Th1 and Th17- mediated immune responses in LPS-induced ALI mice.

\section{Discussion}

In this study, one of the most interesting observations is that LPS-induced ALI resulted in a rapid cDCs accumulation and developed a feature of Th1 and Th17 polarization responses in the lungs. More importantly, our results demonstrated that losartan led to inhibition of respiratory cDCs maturation and impaired Th1 and Th17 immune responses compared with ALI mice. Altogether, these data show that the protective effect of losartan in ALI may be dependent, at least in part, on inhibition of lung cDCs maturation and suppression of Th1 and Th17 polarization responses.

A clinically relevant model of ALI induced by i.t. injection of LPS was used in our experiment, which might mimic sepsisinduced ALI in humans (24). The successful establishment of the LPS-induced ALI mice model made it possible for us to 
A

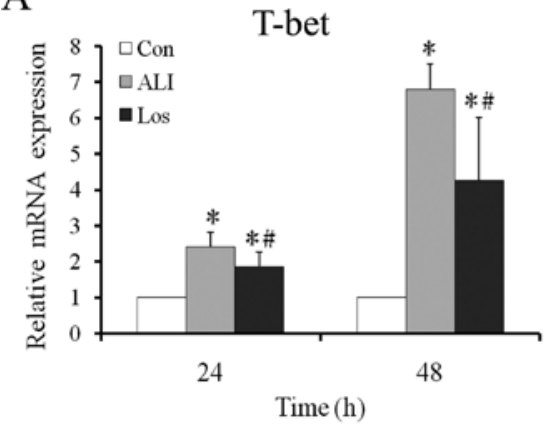

C

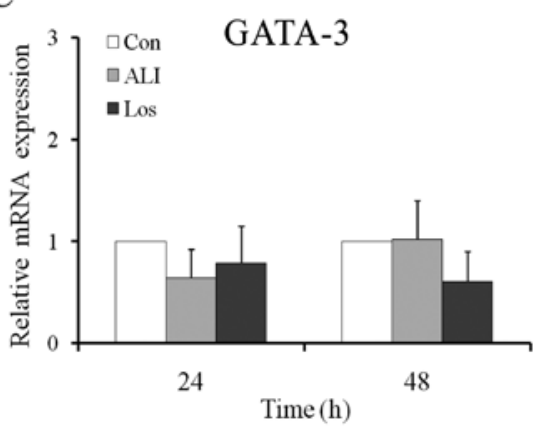

E

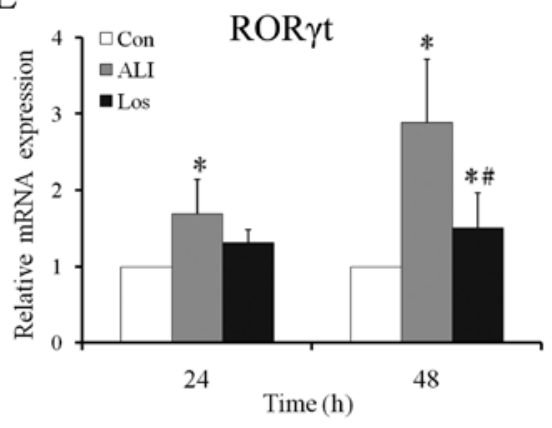

B

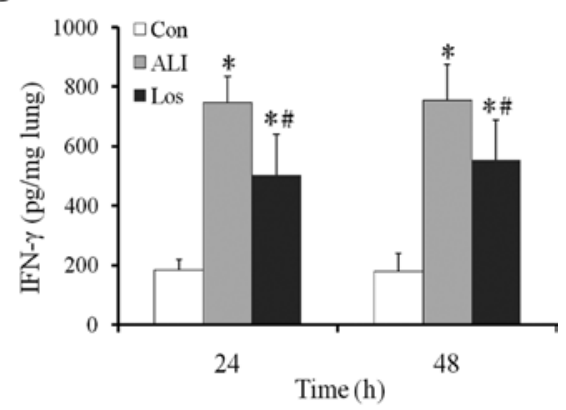

D

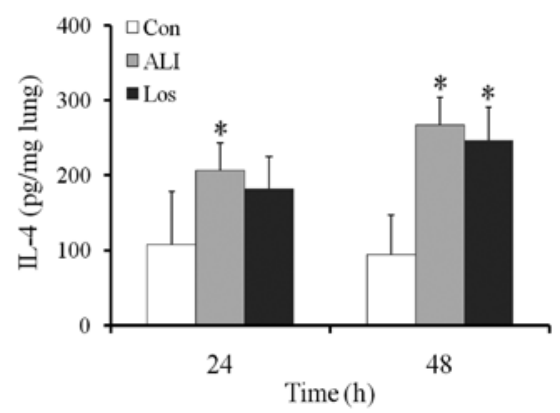

F

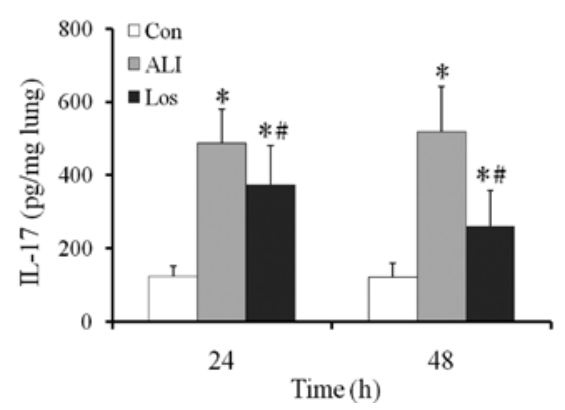

Figure 3. Losartan inhibits Th1 and Th17 polarization responses in ALI mice at 24 and $48 \mathrm{~h}$. Real-time PCR was performed to evaluate the mRNA expression of (A) T-bet, (C) GATA-3 and (E) ROR $\gamma$ t. ELISA was used to assess concentrations of (B) IFN- $\gamma$, (D) IL-4 and (F) IL-17 in lung homogenates. Data are the mean $\pm \mathrm{SD}$ ( $\mathrm{n}=5$ or 6 for each group at 24 and $48 \mathrm{~h}$ ). ${ }^{*} \mathrm{P}<0.05$ vs. the Con mice; ${ }^{*} \mathrm{P}<0.05$ vs. the ALI mice.

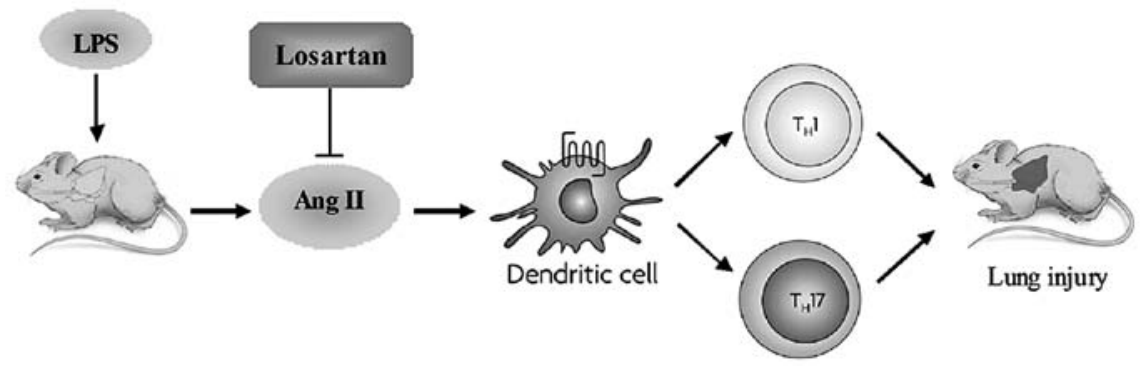

Figure 4. Schematic diagram of the partial protective mechanisms of losartan in LPS-induced ALI mice. Ang II, angiotensin II; LPS, lipopolysaccharide.

test the role of losartan in ALI (Fig. 1). In accordance with previous observations $(3,5)$, we found that the administration of losartan partially prevented lung injury in LPS-induced ALI mice (Fig. 1). Further investigations are needed to elucidate the protective mechanisms of losartan in ALI.

DCs are potent antigen-presenting cells that are crucial for the priming phase of the immune response (6). However, little is known about a role for DCs in ALI. Our results demonstrate that there is a rapid accumulation of cDCs in the lung during the early phase of LPS-induced ALI (Fig. 2). Consistent with our results, a recent study showed that infiltration of numerous DCs played a key pro-inflammatory role in bleomycin-induced lung injury (10). Moreover, Venet et al (32) observed that large amounts of DCs are induced and activated in the lung in a murine model of indirect ALI. These results suggest that the ways to decrease the recruitment of cDCs to the lung or control maturation of DCs may represent new venues in treating this disease. 
Mature or activated DCs are characterized by a further increase of co-stimulatory molecules on their surface (7). CD80, also known as B7-1, is the classic positive co-stimulatory molecule that is expressed on the surface of DCs. The function of MHC II is the presentation of short, pathogen-derived peptides to T cells. Binding of these ligands to receptors is essential for T-cell activation (6). In the present model, our results demonstrated that the accumulated cDCs in ALI mice expressed a more mature phenotype accompanied by increased CD80 molecules (Fig. 2).

Suppression of DC function by some drugs may be essential to prevent or treat ALI. Several in vitro and in vivo studies have demonstrated that losartan inhibits DC activation $(14,15,17)$. Elucidating the effects that losartan has on these factors may provide further insight into the pathology of inflammation, given that these factors are important in the development of LPS-induced ALI. Our data demonstrate that losartan significantly inhibited lung cDCs maturation in LPS-induced ALI mice at $6 \mathrm{~h}$ (Fig. 2). Similar to our results, a recent study demonstrated that bleomycin-induced lung injury is clearly attenuated when using a novel DC inhibitor to inactivate lung DCs (10). We have now shown that losartan is one such drug that can partially block cDCs activation, providing a potential mechanism by which losartan exerts its anti-inflammatory effect and ameliorates LPS-induced lung injury.

Because DCs present antigens so efficiently, they are thought to be critical determinants of Th cell polarization (6). T-helper cells could be divided into subsets: Th1, Th2 and Th17 (11). Th1 cells activate macrophages, and are essential for the defense against intracellular pathogens. Th2 cells are important in recruiting eosinophils to sites of inflammation and helping to clear parasitic infections. Th17 cells are recently-described inflammatory $\mathrm{CD} 4^{+} \mathrm{T}$ cells, which participate in clearing pathogens during host defense reactions and are involved in tissue inflammation $(6,11)$. Imbalance of Th cell subsets has been implicated in the pathogenesis of some inflammatory diseases $(7,33)$.

After induction of ALI, we observed that LPS-induced ALI was characterized by pro-inflammatory Th1 and Th17 immune response in the lung at 24 and $48 \mathrm{~h}$ (Fig. 3). The present findings are consistent with recent study from another laboratory wherein a number of $\mathrm{CD} 4^{+} \mathrm{T}$ cells were recruited to the lung at $24 \mathrm{~h}$ in a murine model of indirect ALI (34). These results are also in agreement with two recent clinical observations of critically ill patients, which show severe H1N1 influenza with ARDS characterized by early secretion of Th17 (IL-6, IL-8, IL-9, IL-17) and Th1 (TNF- $\alpha$, IL-15, IL-12p70) cytokines $(12,13)$. These cytokine profiles have been previously reported to participate in pro-inflammatory responses.

Previous studies have demonstrated that $\mathrm{T}$ cells produce all components of the RAS, and Ang II has direct actions on $\mathrm{T}$ cell function $(15,16,18)$. We postulated that Th cells may be another possible pathway of losartan anti-inflammatory effect on ALI mice. In our model, our results demonstrated that losartan suppressed inflammatory Th1 and Th17 polarization, especially Th1 immune responses (Fig. 3). These observations are supported by recent studies showing that losartan inhibits polarized Th1/Th17-mediated inflammatory diseases $(15,18)$. Our findings extend these previous findings and definitely demonstrated that impaired Th1 and Th17 response caused by losartan in LPS-induced ALI is one of the novel mechanisms. An interesting finding in our present study is that in contrast to unaltered GATA-3 mRNA at $24 \mathrm{~h}$ and $48 \mathrm{~h}$, a significant increase in IL-4 was noted at $24 \mathrm{~h}$ and $48 \mathrm{~h}$ in the LPS-induced ALI mice. Although the exact reason remains unclear, one possibility is that there are several other cellular sources of IL-4, including NK T cells, $\gamma \delta \mathrm{T}$ cells, basophils, mast cells, eosinophils and stromal cells (35).

In summary, our results demonstrate that the protective effect of losartan in ALI may be dependent, at least in part, on inhibition of lung cDCs maturation and suppression of Th1 and Th17 immune response (Fig. 4).

\section{Acknowledgements}

This study was supported by the National Natural Science Foundation of China (project 30640012) and Scientific Research Projects for Postgraduate Students of the Jiangsu Province (CX09B-066Z). We thank Professor Wang Li-Xing, Professor Zhang Jian-Qiong and Professor Shen Chuan-Lai (Southeast University School of Medicine, Nanjing) for generously providing kind advice.

\section{References}

1. Zhang H and Slutsky AS: Year in review 2008: critical care respirology. Crit Care 13: 225, 2009.

2. Imai Y, Kuba K, Rao S, et al: Angiotensin-converting enzyme 2 protects from severe acute lung failure. Nature 436: 112-116, 2005.

3. Kuba K, Imai Y, Rao S, et al: A crucial role of angiotensin converting enzyme 2 (ACE2) in SARS coronavirus-induced lung injury. Nat Med 11: 875-879, 2005.

4. Hagiwara $S$, Iwasaka H, Matumoto S, Hidaka S and Noguchi T: Effects of an angiotensin-converting enzyme inhibitor on the inflammatory response in in vivo and in vitro models. Crit Care Med 37: 626-633, 2009.

5. Liu L, Qiu HB, Yang Y, Wang L, Ding HM and Li HP: Losartan, an antagonist of AT1 receptor for angiotensin II, attenuates lipopolysaccharide-induced acute lung injury in rat. Arch Biochem Biophys 481: 131-136, 2009.

6. Heath WR and Carbone FR: Dendritic cell subsets in primary and secondary $\mathrm{T}$ cell responses at body surfaces. Nat Immunol 10: 1237-1244, 2009.

7. Vermaelen K and Pauwels R: Pulmonary dendritic cells. Am J Respir Crit Care Med 172: 530-551, 2005.

8. Cosio M G, Saetta M and Agusti A: Immunologic aspects of chronic obstructive pulmonary disease. N Engl J Med 360: 2445-2454, 2009.

9. Shinohara ML and Cantor $\mathrm{H}$ : The bridge between dendritic cells and asthma. Nat Med 13: 536-538, 2007.

10. Bantsimba-Malanda C, Marchal-Somme J, Goven D, Freynet O, Michel L, Crestani B and Soler P: A role for dendritic cells in bleomycin-induced pulmonary fibrosis in mice. Am J Respir Crit Care Med 182: 385-395, 2010.

11. Zhu J, Yamane H and Paul WE: Differentiation of effector CD4 T cell populations. Annu Rev Immunol 28: 445-489, 2010.

12. Bermejo-Martin JF, de Lejarazu RO, Pumarola T, et al: Th1 and Th17 hypercytokinemia as early host response signature in severe pandemic influenza. Crit Care 13: R201, 2009.

13. Hagau N, Slavcovici A, Gonganau DN, et al: Clinical aspects and cytokine response in severe H1N1 influenza A virus infection. Crit Care 14: R203, 2010.

14. Nahmod KA, Vermeulen ME, Raiden S, et al: Control of dendritic cell differentiation by angiotensin II. FASEB J 17: 491-493, 2003.

15. Jurewicz M, McDermott DH, Sechler JM, et al: Human T and natural killer cells possess a functional renin-angiotensin system: further mechanisms of angiotensin II-induced inflammation. J Am Soc Nephrol 18: 1093-1102, 2007.

16. Hoch NE, Guzik TJ, Chen W, et al: Regulation of T-cell function by endogenously produced angiotensin II. Am J Physiol Regul Integr Comp Physiol 296: R208-R216, 2009. 
17. Stegbauer J, Lee DH, Seubert S, et al: Role of the renin-angiotensin system in autoimmune inflammation of the central nervous system. Proc Natl Acad Sci USA 106: 14942-14947, 2009.

18. Platten M, Youssef S, Hur EM, et al: Blocking angiotensinconverting enzyme induces potent regulatory $\mathrm{T}$ cells and modulates TH1- and TH17-mediated autoimmunity. Proc Natl Acad Sci USA 106: 14948-14953, 2009.

19. Taooka Y, Maeda A, Hiyama K, Ishioka S and Yamakido $M$ Effects of neutrophil elastase inhibitor on bleomycin-induced pulmonary fibrosis in mice. Am J Respir Crit Care Med 156: 260-265, 1997.

20. Smith KM, Mrozek JD, Simonton SC, Bing DR, Meyers PA, Connett JE and Mammel MC: Prolonged partial liquid ventilation using conventional and high-frequency ventilatory techniques: gas exchange and lung pathology in an animal model of respiratory distress syndrome. Crit Care Med 25: 1888-1897, 1997.

21. Koya T, Kodama T, Takeda K, et al: Importance of myeloid dendritic cells in persistent airway disease after repeated allergen exposure. Am J Respir Crit Care Med 173: 42-55, 2006.

22. Cleret A, Quesnel-Hellmann A, Vallon-Eberhard A, et al: Lung dendritic cells rapidly mediate anthrax spore entry through the pulmonary route. J Immunol 178: 7994-8001, 2007.

23. Kiwamoto T, Ishii Y, Morishima Y, et al: Transcription factors T-bet and GATA-3 regulate development of airway remodeling. Am J Respir Crit Care Med 174: 142-151, 2006.

24. Matute-Bello G, Frevert CW and Martin TR: Animal models of acute lung injury. Am J Physiol Lung Cell Mol Physiol 295: L379-L399, 2008.

25. von Wulffen W, Steinmueller M, Herold S, et al: Lung dendritic cells elicited by Fms-like tyrosine 3-kinase ligand amplify the lung inflammatory response to lipopolysaccharide. Am J Respir Crit Care Med 176: 892-901, 2007.

26. de Heer HJ, Hammad H, Kool M and Lambrecht BN: Dendritic cell subsets and immune regulation in the lung. Semin Immunol 17: 295-303, 2005.
27. Beaty SR, Rose CE Jr and Sung SS: Diverse and potent chemokine production by lung CD11 $\mathrm{b}^{\text {high }}$ dendritic cells in homeostasis and in allergic lung inflammation. J Immunol 178: 1882-1895, 2007.

28. McWilliam AS, Nelson D, Thomas JA and Holt PG: Rapid dendritic cell recruitment is a hallmark of the acute inflammatory response at mucosal surfaces. J Exp Med 179: 1331-1336, 1994.

29. Osterholzer JJ, Ames T, Polak T, et al: CCR2 and CCR6, but not endothelial selectins, mediate the accumulation of immature dendritic cells within the lungs of mice in response to particulate antigen. J Immunol 175: 874-883, 2005.

30. Grayson MH, Ramos MS, Rohlfing MM, et al: Controls for lung dendritic cell maturation and migration during respiratory viral infection. J Immunol 179: 1438-1448, 2007.

31. Kabir K, Gelinas JP, Chen M, et al: Characterization of a murine model of endotoxin-induced acute lung injury. Shock 17: 300-303, 2002.

32. Venet F, Huang X, Chung CS, Chen Y and Ayala A: Plasmacytoid dendritic cells control lung inflammation and monocyte recruitment in indirect acute lung injury in mice. Am J Pathol 176: 764-773, 2010.

33. Xia N, Zhou S, Liang Y, et al: $\mathrm{CD}^{+} \mathrm{T}$ cells and the Th1/Th2 imbalance are implicated in the pathogenesis of Graves' ophthalmopathy. Int J Mol Med 17: 911-916, 2006.

34. Venet F, Chung CS, Huang X, Lomas-Neira J, Chen Y and Ayala A: Lymphocytes in the development of lung inflammation: a role for regulatory $\mathrm{CD} 4^{+} \mathrm{T}$ cells in indirect pulmonary lung injury. J Immunol 183: 3472-3480, 2009.

35. Nelms K, Keegan AD, Zamorano J, Ryan JJ and Paul WE: The IL-4 receptor: signaling mechanisms and biologic functions. Annu Rev Immunol 17: 701-738, 1999. 